\title{
Transformaciones del modelo territorial de Santiago de Chile
}

\author{
Jonás Figueroa Salas
}

\section{Resumen}

La identificación de las tendencias morfológicas, como expresiones espaciales del crecimiento físico del Área Metropolitana de Santiago de Chile, sirve para reflexionar acerca de las diferentes y variadas contradicciones -negaciones y discontinuidadesque operan eventualmente entre el modelo territorial propuesto por los instrumentos de ordenamiento y la planta final resultante. Junto a ello, esta reflexión nos brinda la oportunidad de identificar las tendencias registradas por una urbanística de escala metropolitana, cuyo signo de los tiempos es el todo urbanizable, dejando de lado otras maneras y modos de encarar el crecimiento físico de la ciudad. En tal sentido, el diseño de una armadura de escala provincial, como factor de reequilibrio entre un débil sistema regional de ciudades y un área metropolitana excedida, constituye la segunda parte del escrito.

Palabras claves: MODELOS TERRITORIALES, MORFOLOGÍA URBANA, CRECIMIENTO FÍSICO, PLANIFICACIÓN URBANA, POLÍTICAS PÚBLICAS, ORDENAMIENTO TERRITORIAL, TALAGANTE, SANTIAGO DE CHILE. MICROREGIÓN DE SANTIAGO DE CHILE.

Filiación: El Arqto. Jonás Figueroa Salas es profesor titular e investigador en la Universidad de Santiago de Chile.

\section{Índice}

Introducción

1. Modelo territorial propuesto por la planificación intercomunal

2. Modelo territorial resultante en el horizonte de 30 años

3. Modelo territorial alternativo

3.1. Estrategias territoriales de inicio de siglo XXI

3.2. La provincia como dimensión de reequilibrio regional

3.3. Hacia un nuevo modelo territorial de escala provincial

3.3.1. El modelo provincial vigente

3.3.2. Objetivos y criterios de ordenamiento

3.3.3. El modelo provincial propuesto

4. A modo de conclusión

Referencias 


\section{I ntroducción}

En las tres últimas décadas del siglo XX, Chile ha vivido dos procesos políticos (1973 y 1989), que se han expresado en senda reformas de la armadura administrativa del país. A pesar de los grandes impulsos iniciales que ha dado la autoridad política a estos procesos, los nuevos ordenamientos no se han traducido en cambios significativos en la arquitectura ni en la estructura del territorio.

El proceso de organización administrativa que se formula y ejecuta bajo la dictadura militar, a mediados los años setenta, organiza el país en 13 regiones y 54 provincias, duplicando las 25 iniciales. Después de algo más de 30 años de andadura, este proceso de regionalización se nos muestra con resultados desiguales, en donde los grandes problemas que justificaron su formulación persisten $y$, en algunos casos, se han agravado. El privilegio que han tenido, por ejemplo, los asuntos geopolíticos y también los productivos sobre factores de equidad territorial, han debilitado el proceso, asistiéndose en la actualidad hacia una estrategia que busca redimensionar la superficie de las regiones mediante la división y aumento del número de ellas, sin llegar a actuar en cuestiones relacionadas con el ordenamiento territorial de estas entidades mayores, con el fin de incorporar mejoras sustantivas en sus capacidades y oportunidades. De esta forma, vemos por ejemplo, que de las tres regiones posibles de constituir dimensiones metropolitanas (Santiago, Valparaíso y Concepción), se reconoce sólo una como tal, Santiago. Mientras tanto, la Región de Valparaíso apenas constituye un conjunto de núcleos de población organizado linealmente a lo largo de la cuenca y el trazado del río Aconcagua. Ni siquiera la anexión del litoral históricamente perteneciente a la Región de Santiago ha venido a introducir modificaciones en la ocupación equilibrada del territorio regional ${ }^{1}$. El caso de Concepción registra situaciones de aglomeración más parecidas a la región capital de Santiago y en donde los núcleos originales tienden a la conformación de una única extensión urbana y un hinterland prácticamente inexistente y disperso.

El posterior retorno a la institucionalidad democrática, a partir de los años 90 del siglo $X X$, acentúa su atención más en la escala municipal que en la regional. En ambas situaciones, las provincias han pasado a constituir entes debilitados y de imprecisa participación en la organización política del país. Procesos, todos ellos, que parecen más bien estrategias de una idea desconocida de país, carentes de razones y sin un norte que lo dirija hacia una nación moderna e integrada. Cuestión que se suma a la inexistencia de un cuerpo teórico en la materia y la falta de debate de naturaleza pública, académica o parlamentaria que lo alimente.

A esta debilidad en materias territoriales, se agregan también las debilidades metodológicas que exhibe la urbanística chilena, tanto en el plano conceptual como en el instrumental. Ambas cuestiones se encuentran motivadas entre otras, porque las principales determinaciones y normativas de la planificación urbana no responden a un esquema o modelo territorial que supere los conflictos que registra el modelo tendencial vigente caracterizado por una exacerbado centralismo, que se repite tanto en las escalas nacional, regional y local, pasando ello a ser una de los principales obstáculos.

En una primera fase, el modelo territorial es un esquema teórico que debería expresar la voluntad de la ciudadanía dada a conocer a través de sus representantes, la

\footnotetext{
${ }^{1}$ Al respecto puede verse Pávez R., M. Isabel: "Identidad, ordenamiento territorial y oportunidades: espacios públicos y recreación para la Región Metropolitana de Santiago Siglo XXI", en REVISTA DE URBANISMO, digital, N¹4, D. Urbanismo F.A.U. U. de Chile, Primer semestre 2006, 21 págs. ilustradas. $<$ http://revistaurbanismo.uchile.cl>
} 
valoración de los atributos y la corrección de los conflictos. Estos tres insumos básicos son necesarios para conocer las tendencias, limitaciones y oportunidades de una determinada dimensión espacial. En fases posteriores del proceso de formulación del instrumento urbanístico, el modelo interviene hipotéticamente estos signos a través de criterios de ordenamiento y operaciones estratégicas y los pone al servicio de un proyecto territorial.

Surgido como síntesis de la expresión física del diagnóstico y como un ejercicio de comprensión de atributos y conflictos identificados durante las fases de recogida y procesamiento de información, el modelo territorial se nos presenta como una referencia conceptual a tener presente en la redacción del instrumento de ordenamiento.

De este modo, el plan regulador intercomunal respondería no sólo a las tendencias, sino también a un proyecto posible de ciudad y de territorio. Al esquema conceptual que expresa gráficamente el diagnóstico, lo denominaremos indistintamente tendencial, vigente o problema. Al esquema de referencia que sintetiza las principales operaciones estratégicas que transforman los conflictos en oportunidades de cambio y los atributos en signos de desarrollo, lo denominaremos indistintamente modelo propuesto o simplemente proyecto urbano o territorial, de acuerdo con la escala espacial sobre la que interviene.

Este artículo esboza algunas de las caracterizaciones sobre los cuales es posible asentar una reflexión acerca de los alcances de los instrumentos de ordenamiento de la gran escala y también, formular operaciones estratégicas que permitan plasmar un proyecto territorial de escala provincial (microregional) en oposición alternativa al modelo territorial que implanta el Ministerio de Vivienda y Urbanismo (MINVU), que confunde o asimila la escala metropolitana con la regional. Así, frente a la desintegración física y funcional que actualmente registra el sistema regional de ciudades, la integración surge como una de las estrategias posibles de un nuevo modelo a partir del reconocimiento de sus partes.

Siguiendo a Serrano ${ }^{2}$, entendemos por modelo territorial la forma y los modos que utiliza una sociedad para ocupar y transformar un espacio determinado, en su doble carácter de proceso acumulativo y social. El sistema de ciudades y sus áreas de influencia asociada, y las relaciones e interrelaciones laborales, económicas, de servicios y sociales desempeñan un papel relevante a la hora de definir un determinado modelo territorial.

\section{Modelo territorial propuesto por la planificación intercomunal}

EI Plan Microregional de Santiago (PMRS - MOP 1960, IV-B o Microregión de Santiago ${ }^{3}$ ), constituía el referente administrativo y el marco regional del Plan Intercomunal de Santiago (PRIS - MOP 1960-1994). También es el documento que define las variables de población, localización y las relaciones sistémicas que se establecen a partir de ello, en el espacio microregional.

\footnotetext{
2 SERRANO, Antonio, “EI modelo territorial europeo: tendencias para el siglo XXI y sus implicaciones para el modelo territorial español", en revista URBAN № 8 Madrid, editada por la ETSAM - UPM, otoño 2003, pp. 35 $-54$.

${ }^{3}$ Véase al respecto CHILE, MINISTERIO DE OBRAS PÚBLICAS, Dirección de Planeamiento, Departamento de Planes de Obras Públicas, BRIEVA ALVARADO, Amador y GONZÁLEZ MONTECINOS, Ventura [autores en 1958], La Unidad Micro-Regional: características y métodos para delimitarla. Santiago de Chile, Publicación N6, junio de 1962, documento citado por Pávez R., M. I., 2006, op. cit. infra, p. 170.
} 
El debate que surge al alero de la formulación del primer plan de urbanismo que se propone ordenar un crecimiento físico que se expande más allá de los ámbitos municipales y departamentales, se encuentra lleno de ricas referencias que emparentan los planteamientos teóricos que antecedieron a la aprobación y promulgación del PRIS, con aquellos que se debatían en la Europa de pre y postguerra del siglo $X X$.

Gran parte de estas referencias conocidas en el ámbito local a través de documentos académicos y publicaciones técnicas, consisten en planteamientos conceptuales e instrumentales que por las vicisitudes bélicas europeas carecían de datos precisos acerca de la evaluación de sus resultados, al no haber sido ejecutados ni siquiera parcialmente. Antecedentes que hubiesen permitido matizar en el plano local, unas ideas de ciudad y de región que hasta ese momento tenían un fuerte sesgo teórico.

La propuesta de definir el modelo territorial basado en la identificación de núcleos urbanos integrantes de un sistema de ciudades menores con roles claros en la economía regional y relaciones de dependencia en diversos grados con la gran ciudad según su lejanía a ella, intentaba una forma de reorganizar la microregión con el objetivo de volcar los futuros crecimientos de la metrópolis en ella, a través de la desconcentración funcional y socioeconómica Santiago, alejándose expresamente del modelo de desarrollo disperso que comenzaba a operar con los altos costos sobre la matriz geográfica agrícola y la extensión de redes. Cabe destacar que los roles asignados miraban especialmente las potencialidades locales incluyendo los signos de valor histórico y cultural, aunque esto se producía desde el nivel central de la administración y sin participación de la población local ${ }^{4}$.

Sin embargo, la administración del presidente Eduardo Frei Montalva (1964-1970) junto con priorizar los megaconjuntos residenciales y de equipamientos complementarios para la clase media - proyectos y clase social emblemáticos a los cuales los habitantes más pobres debían aspirar- en el pericentro de Santiago (Remodelación San Borja, por ejemplo), formula políticas que promueven la descentralización de Santiago en la "Macroregión Central de Chile" (especialmente en Rancagua y Valparaíso), saltándose la escala y las oportunidades que ofrece la propia microregión de Santiago 5 .

Más adelante, en los estudios para la región central de Chile llevados a cabo a comienzos de los años 70, los satélites para la desconcentración de Santiago más próximos analizados, presentaban, en principio, índices relativamente favorables en el rango de costos, pero se concluyó finalmente que no convenía orientar hacia ellos el crecimiento de la ciudad, pues, para alcanzar el rango favorable en costos de urbanización, deberían absorber una parte tan considerable del crecimiento metropolitano, que su crecimiento tendría que ser incentivado, acarreando inevitablemente el fracaso de la política desconcentradora global ${ }^{6}$.

\footnotetext{
${ }^{4}$ PÁVEZ REYES, María Isabel. 2006. Vialidad y Transporte 1950-1979 en Santiago de Chile: Concepto y Estrategia de Ordenación del Territorio en el marco de la Planificación Urbana - Regional por el Estado de Chile. Tesis Doctoral en Arquitectura y Urbanismo, Universidad Politécnica de Madrid, Director Dr. Ing. Julio Pozueta E., 13 de diciembre de 2006.

5 RAPOSO, Alfonso; VALENCIA, Marco, 2004. “Modernidad, Diseño Urbano y Utopía: notas sobre el fundamento político de las acciones de Remodelación Urbana en Santiago. El caso de CORMU 1966-1973". Revista de Urbanismo, digital, [en línea: http://revistaurbanismo.uchile.cl] (Primera Parte, en $\mathrm{N}^{\circ}$ 9; Segunda Parte, en №10), Departamento de Urbanismo, FAU. Universidad de Chile.

${ }^{6}$ CIDU, Equipo Macrozona Central,"Síntesis del estudio "Región central de Chile: perspectivas de desarrollo" (1971-1972), en: de MATTOS, C., FIGUEROA, O.; BANNEN, P.; CAMPOS, D. Editores) Santiago en EURE:
} 
La agenda del Seminario de Santiago, realizado en 1990, no consideró incorporar el ordenamiento de la microrregión de Santiago en la actualización del plan intercomunal en curso ${ }^{7}$.

El modelo territorial formulado por el PMRS identifica así, las localidades integrantes del sistema urbano microregional a modo de ciudades satélites. Estos se organizan en función de tres anillos, situados en un radio de 15, 30 y $45 \mathrm{Km}$. en torno a la metrópolis. La función productiva de naturaleza industrial define la primera corona regional (situadas entre los 13 y $20 \mathrm{~km}$. del núcleo central; de carácter agrícola, las localidades situadas en la segunda corona situada entre los 18 y 38 km.; y polifuncional, aquellas que se sitúan más allá del tercer anillo regional, entre los 38 y los $60 \mathrm{~km}$. Más allá de su grado de dependencia y de influencia, todas estas localidades fueron consideradas inicialmente e indiferentemente como núcleos satélites del núcleo mayor. (Ver Fig. 1)

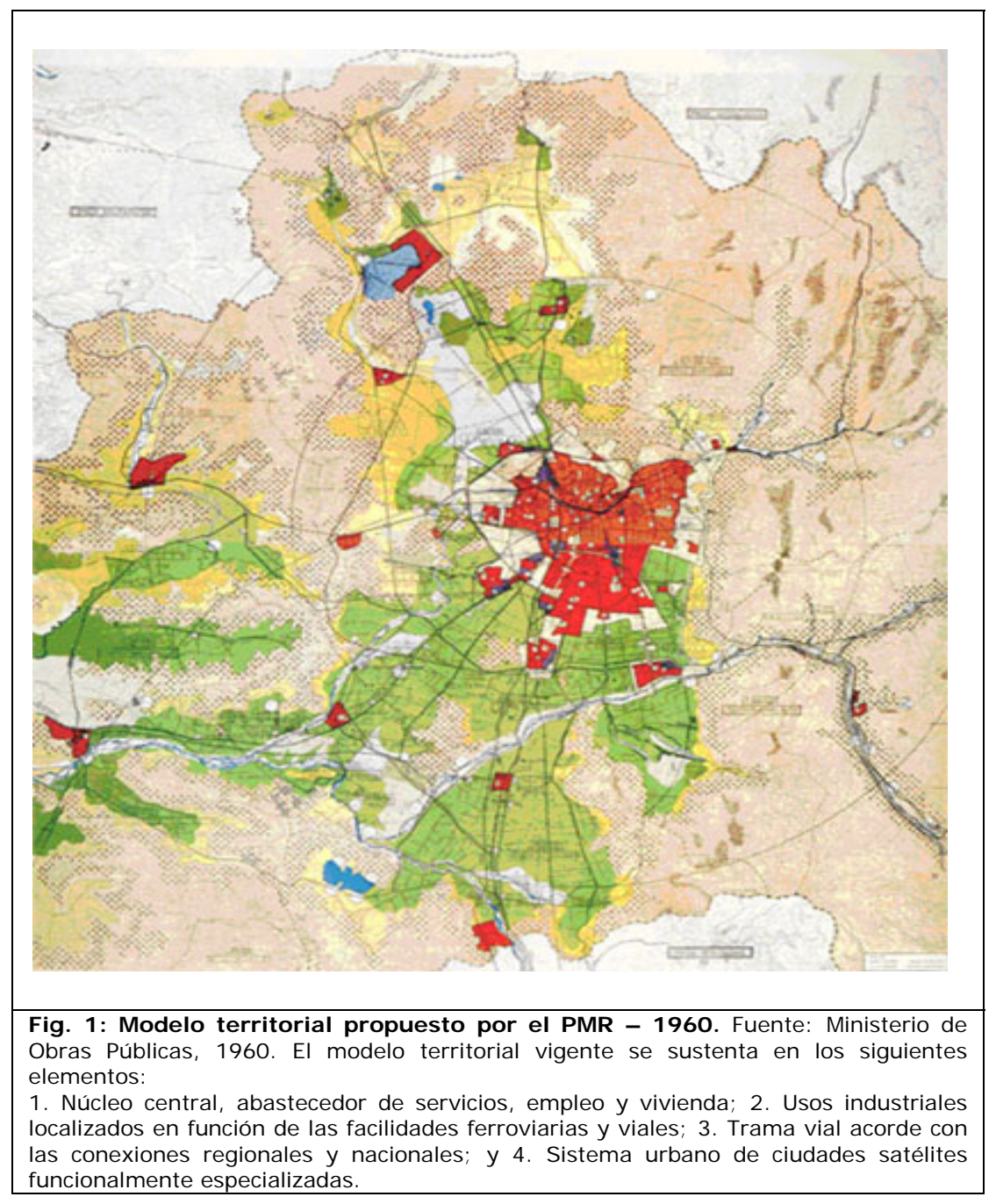

Huellas de una metamorfosis metropolitana 1970/2000, I.E.U.y T., P. Universidad Católica de Chile, 545 págs.

7 Ponencia de M. I. Pávez R. en reuniones preparatorias del "SANTIAGO SIGLO XXI" MINVU, (Edificio Diego Portales, dic. 1991) realizadas en Casa de la Cultura Parque Metropolitano San Cristóbal. En: DOC UR N³37, Ed. Departamento de Urbanismo, FAU, U. de Chile). 


\section{Modelo territorial resultante}

Diversas limitaciones, tales como el abandono de parte de los organismos competentes para elaborar un plan de acciones que hubiese plasmado el modelo propuesto y la falta de estrategias precisas en la materia condicionan la concreción de la organización espacial formulada por el Plan Microregional. Antes del plan, estas localidades exhiben un funcionamiento y una espacialidad propia de pequeñas localidades fuertemente relacionadas en su desarrollo con las actividades productivas de su entorno rural. Es así que San Bernardo y Maipú, como exponentes de estos satélites, registran un peso importante relacionado con lo ferroviario y lo agrícola, por ejemplo. En el horizonte de los 20 y 30 años de vigencia del Plan Intercomunal de Santiago de 1960, estas localidades satélites han sido anexionadas al continuo urbano de escala metropolitana, como ciudades dormitorios que responden a las demandas provenientes de la ciudad central.

Las localidades satélites de la primera corona regional fueron rápidamente anexadas al núcleo central por su propio crecimiento. Anexión física que constituye la principal alteración que experimenta el PRIS y que es reforzada por las otras anexiones que a partir de ello se producen de modo espontáneo. Anexión física, derivada de anteriores anexiones funcionales, anexión administrativa hasta culminar el año 1994 con la anexión urbanística por la incorporación de estas localidades al Plan Regulador Metropolitano de Santiago (PRMS - 1994), que actualiza el original PRIS de 1960.

Más que resolver los temas relacionados con el ordenamiento de su propio desarrollo urbano, la incorporación de las provincias, algunas de ellas carentes de una propia estructura y de un propio armazón físico, a esquemas urbanos que observan problemas, déficits y oportunidades de orden y escala metropolitana, no es la estrategia más afortunada para resolver los problemas de dependencia y retraso que registra el sistema regional. Antes bien, se desconoce el problema y se introducen otros, tales como aparición de modalidades de ocupación y crecimiento demográficos externos a la propia localidad provincial.

Esta anexión física junto a otras anexiones de orden funcional, administrativo y urbanístico de las localidades situadas dentro del territorio que dibujaba el anillo de 15 $\mathrm{km}$ que, por su histórica dependencia funcional, anterior a la propia formulación del PRIS, son rápidamente anexadas por un crecimiento físico que se desplaza utilizando la vialidad radial como principal vehículo del crecimiento, ha sido un fenómeno continuo en los últimos 50 años, transformado los tres principales núcleos del primer anillo regional -Maipú, San Bernardo y Puente Alto- en núcleos de mayor importancia poblacional y económica que un buen número de capitales regionales y provinciales, pasando de tener, al inicio del período (1960) unos pocos miles de habitantes, a cientos de miles al final del mismo (2000). (Ver Fig. 2). 


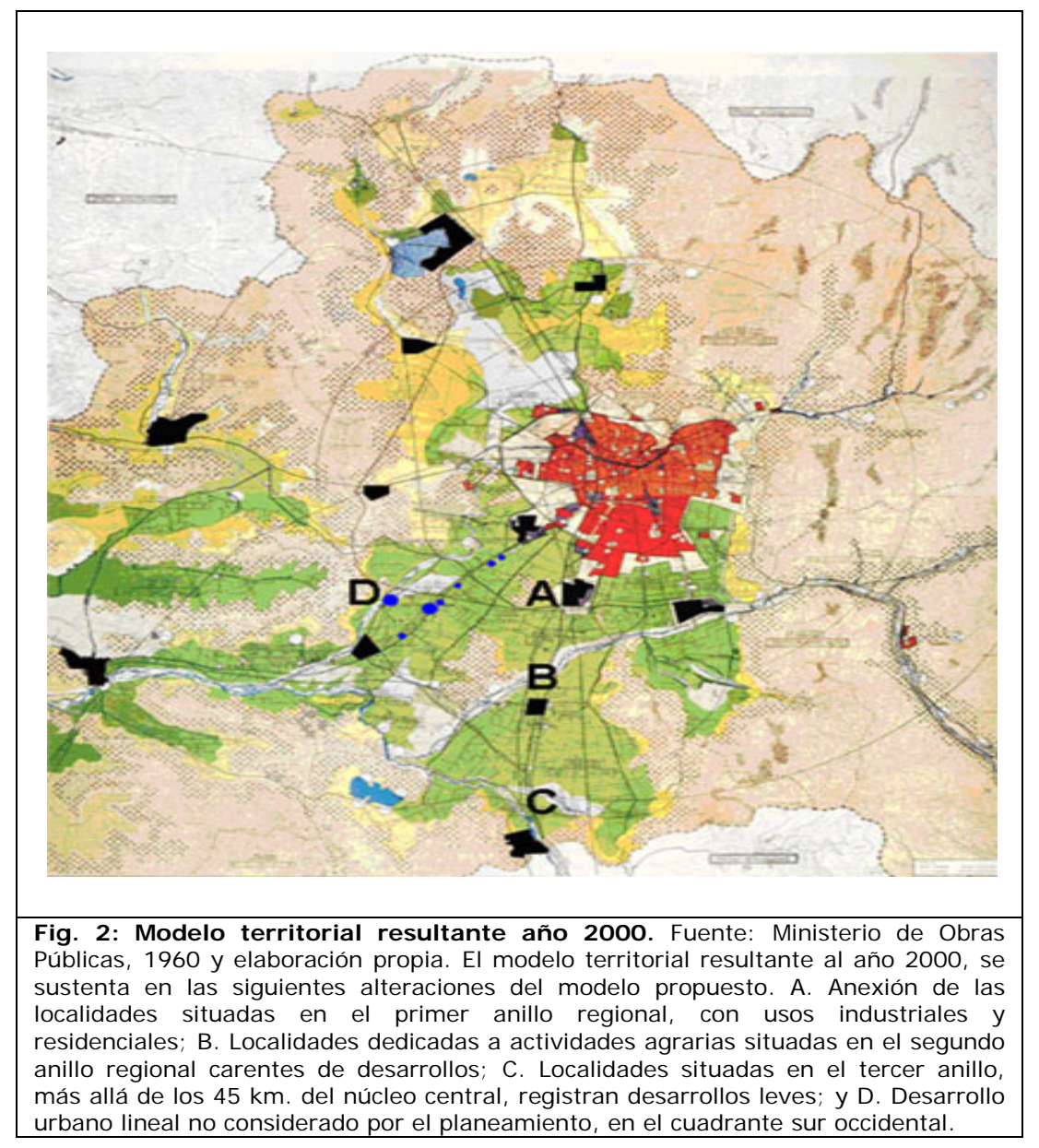

\section{Modelo territorial alternativo}

\subsection{Estrategias territoriales de inicio de siglo XXI}

Gran parte de la inversión pública que hoy se localiza en el corredor suroccidental de la Región Metropolitana, en donde se emplaza la provincia de Talagante, una de las seis que configuran la Región Metropolitana, está vinculada a la introducción de mejoras en las facilidades viales. Circunstancia que, de paso, altera radicalmente la imagen de marginación de los procesos de desarrollo urbano de origen metropolitano que identifica a las localidades situadas en este corredor suroccidental. La entrada en funcionamiento de la llamada "Autopista del Sol" (al puerto de San Antonio) ha anulado de plano el caótico espacio periférico metropolitano, de naturaleza agrícola, industrial y habitacional de baja calidad urbana, que anteriormente separaba el valle de Talagante con la gran ciudad.

Sin embargo, a pesar de estas oportunidades infraestructurales la carencia de un proyecto de ordenamiento territorial transforma las mismas en oportunidades vacías de contenidos. Hoy en día, Santiago y esta provincia se sitúan en una proximidad que depara no pocas incertidumbres y conjeturas acerca de qué tipo de desarrollo promoverá esta vecindad. 
Es preciso, entonces, formular estrategias para dotar a este espacio provincial de los elementos jurídicos y administrativos necesarios que ordenen el desarrollo inmobiliario y productivo que llegará de la mano de las inversiones infraestructurales y productivas.

La modificación del Plan Regulador Comunal es, por cierto, una oportunidad para razonar sobre el futuro posible de Isla de Maipo a partir de las oportunidades y amenazas que provienen de los grandes cambios que ha comenzado a experimentar el espacio regional metropolitano, durante los años noventa y que, es previsible, se reproduzca por diversas razones durante la próxima década. El desarrollo asociado a condicionantes externas que hoy comienza a manifestarse en el valle, puede expresarse de dos formas: que comparezca a modo de franja de usos de gran intensidad junto al eje vial del corredor (Autopista del Sol) y como ocupaciones dispersas carentes de orden y concierto, sin un claro ordenamiento. En ambas situaciones, y por razones de accesibilidad y conexión, Isla de Maipo es el espacio comunal menos beneficiado. Es más, esta comuna podría incrementar el carácter de rinconada a la cual se llega y se retorna, que se encuentra presente en el imaginario del habitante metropolitano más dado a los circuitos, que a relaciones lineales de ida y vuelta.

Entonces, es necesario formular estrategias que transformen las debilidades generadas por el emplazamiento excéntrico y perimetral de Isla de Maipo, en fortalezas. Estas estrategias en el peor de los casos, deberán inducir el surgimiento de piezas de desarrollo alternativo y productos de consumo diversificado a aquellos que por naturaleza surgirán junto a la franja del eje vial de la provincia. De este modo, Isla de Maipo se asociará a otro escenario posible y alternativo de los que se emplazan con pocas diferenciaciones junto al corredor metropolitano.

También, estos desarrollos promovidos por las fuerzas regionales y metropolitanas se deberán expresar en la comuna de Isla de Maipo como áreas de actuación diferenciadas. Junto a las nuevas áreas demarcadas por los desarrollos de origen interior, deberán formularse otras que atiendan los desarrollos motivados por desencadenantes externas.

En el Corredor Metropolitano Suroccidental comparecen factores altamente comprometidos con el futuro, tanto de las propias localidades emplazadas en éste como de la metrópolis y la región. Estos factores se encuentran relacionados con las posibilidades de promover un desarrollo en donde los nuevos usos urbanos no atenten en contra de las actividades productivas agrarias tradicionales. Por el contrario, este desarrollo propuesto y aplicado por un instrumento de regulación y ordenamiento urbanístico debe formular nuevos códigos de una urbanidad complementaria y compatible con las actividades productivas tradicionales. El segundo aspecto se deriva del primero y dice relación con la oportunidad que se presenta a los técnicos y autoridades para explorar nuevos conceptos e instrumentos operativos, acorde con una urbanidad emplazada en un paisaje y en una cultura rural. En tal situación, lo urbano no se expresa a la manera de una ciudad consolidada, sino como una dimensión física situada en medio de una ruralidad que la concibe y sustenta.

Pensar el rol territorial de la provincia de Talagante transciende el hecho que lo motiva. También, constituye una oportunidad para explorar nuevas modalidades de cultura urbanística y de innovaciones metodológicas en el hacer de la planificación y el ordenamiento territorial. 
El plan regulador sea éste de escala comunal o metropolitana, define en primer lugar las modalidades de producción urbana del suelo, amén de ser un instrumento administrativo que formula la organización espacial de los usos del suelo. Atendiendo a ello, consideramos que las normas y ordenanzas como contenidos fundamentales de este tipo de planificación deben ser complementadas con otras figuras conceptuales que tengan el dominio sobre el resultado final: la ciudad. En tal sentido, el proyecto urbano esboza la estructura morfológica del espacio sobre el cual actúa el plan regulador. Entendemos por estructura morfológica las formas que adoptan los principales elementos constituyentes de la estructura urbana: sistema vial; sistema de piezas o paños de borde o perímetro, centralidad, elementos articuladores entre los diferentes usos del suelo; etc.

Entre las grandes vías de conexión regional y nacional hacia el borde litoral central y el sur del país, se emplazan cinco comunas que enlazan la ciudad de Santiago con el territorio agrícola y marítimo emplazado en el corredor suroccidental de la Región Metropolitana. Estas cinco comunas configuran lo que podríamos denominar la corona rural de la gran metrópolis. Padre Hurtado, Peñaflor, Talagante, El Monte e Isla de Maipo, comunas que forman la provincia de Talagante, de acuerdo al Censo del año 1992, reunían una población cercana a las 167 mil personas, alcanzando a cubrir apenas el 3\% del total de habitantes de la Región Metropolitana. Hoy en día se estima que esta población asciende a unas 192 mil personas y al año 2005, se espera que se eleve a unas 220 mil personas.

Las localidades urbanas de Talagante registran un lento poblamiento y bajos crecimientos demográficos vegetativos (sólo 33 mil personas en algo más de una década). Las nuevas cercanías que de modo ingente surgen a raíz de las nuevas ofertas de vialidad rápida de nivel regional, transforman en espacios susceptibles de acoger población y actividades económicas, deslocalizadas desde la metrópolis. Estas oportunidades de nuevos emplazamientos, transforman la provincia en un territorio que podría experimentar un proceso acelerado de cambios, alterando radicalmente su condición de perímetro rural para llegar a constituirse en una espacialidad del tipo dormitorio, que altere la propia imagen que algunas de ellas como Isla de Maipo, aún retienen. Es este un proceso de cambios abierto e incierto que puede consolidar el rol perimetral del sistema urbano y económico del corredor suroccidental y transformar el desarrollo en un inquietante listado de efectos económicos y ambientales perniciosos.

Como ya se ha experimentado en otros espacios regionales dependientes de la centralidad metropolitana, el desarrollo puede comparecer en la provincia destruyendo las particularidades y los atractivos que, como valores diferenciados, existían con anterioridad al fenómeno. En el caso del corredor metropolitano suroccidental, que es lo mismo que decir provincia de Talagante, comparecen situaciones y elementos naturales y construidos que podrían revertir estas amenazas en oportunidades que iluminen cambios positivos.

\subsection{La provincia como dimensión de reequilibrio regional}

El sistema urbano de la provincia de Talagante se encuentra espacialmente articulado sólo por el eje vial del corredor, desconociendo los articuladores naturales que como los ríos Mapocho y Maipo, condicionaron primitivamente su emplazamiento. La localización excéntrica que registra Isla de Maipo con respecto al corredor, se transforma en el principal obstáculo para definir estructuras y tejidos morfológicos de desarrollo propios. Estos estructuras y tejidos siempre serán dependientes de la gravitación funcional y económica que ejerce la metrópolis, más propensa a configurar 
estructuras radiales/lineales, que de tipo reticular, tipo de estructura y tejido que beneficiaría a Isla de Maipo.

La semántica territorial y geográfica que surge del propio razonamiento del rol que se espera que asuma en el futuro la provincia de Talagante en general, y la comuna de Isla de Maipo en particular, comienza a expresarse por pura lógica prospectiva en trampas conceptuales que aprisionan los contenidos proyectuales, y los reducen a simples propósitos y objetivos convencionales. Por otra parte, desde la simplicidad de las imágenes, hoy en día la comuna de Isla de Maipo es un lugar interior situado a orillas de un río que pasa sin promover ningún signo y con una sola calle que va a ninguna parte. Pensar el rol de una determinada pieza o entidad espacial, también implica asumir la imagen cierta o equivocada que de ella cultiva ya sea el propio habitante o ya sea el visitante.

La viabilidad del proyecto territorial de Isla de Maipo pasa por su inserción en el tejido funcional y económico de la provincia de Talagante. Pero, esta inserción debe estar comprometida con determinadas variables que diferencien los productos y facilidades que la comuna proporcione al conjunto provincial y, por extensión al mercado de consumo metropolitano y regional. Por ejemplo, como espacio articulador entre el corredor suroccidental y el corredor sur del valle central, o bien, entre este último y el corredor metropolitano occidental hacia las facilidades turísticas y recreativas del conurbano de Valparaíso - Quintero y Valparaíso - Quillota. En cuanto espacio terminal, este producto diferenciado está fuertemente vinculado a las facilidades paisajísticas e hidrológicas que posee la comuna, sin desconocer las posibilidades patrimoniales artísticas que pueden ofrecer, por ejemplo, unas determinadas tipologías arquitectónicas y constructivas que se asientan en el uso del alero, de la galería cubierta, etc., de las viviendas entre rurales y urbanas que es posible observar en los núcleos habitados. También, en la producción vinífera y frutícola podrían emplazarse otros productos que, como denominaciones de origen (vinos del Valle del Maipo, uva del Maipo, por ejemplo), se constituyan en signos de identidad de la provincia. Hoy en día, éstas últimas son toponimias inciertas. (Ver Fig. 3).

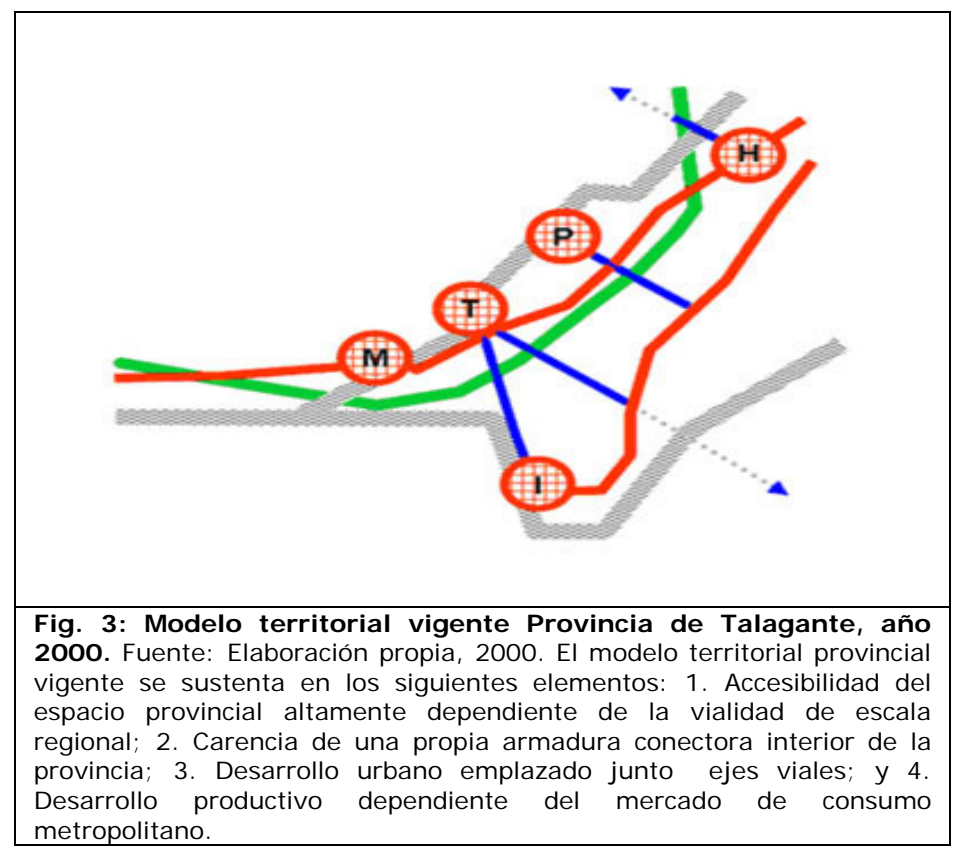




\subsection{Hacia un nuevo modelo territorial de escala provincial}

3.3.1. El modelo provincial vigente. La lectura de las variables que se derivan del emplazamiento de Talagante en el espacio extrametropolitano y regional, señala algunos datos diferenciadores que constituyen los elementos fundamentales del modelo territorial vigente. Estos elementos fundamentales son los siguientes:

La provincia de Talagante constituye un espacio de articulación y transición por encontrarse situado en el corredor que conecta la gran aglomeración metropolitana con las facilidades portuarias y turísticas que posee el litoral central. La identificación de este signo de articulación constituye una pieza fundamental del discurso propositivo, a tener presente en un supuesto proyecto de desarrollo provincial. Ahora bien, la introducción de modificaciones en la estructura vial provincial, a raíz de la nueva condición de la Ruta 78 y su cambio de denominación por la Ruta del Sol (sobrepuesta a la primera), a nivel provincial ha acortado las distancias con la centralidad metropolitana y ha promovido una virtual división del espacio geográfico y funcional de la provincia. Esta división artificial se viene a sumar a las divisiones o separaciones espaciales naturales que generan las topografías y los cuerpos de agua en el tejido provincial: un eje o corredor urbano por el norte y una localidad aislada, por el sur.

Mas, tampoco podría asegurarse que las principales localidades emplazadas en el corredor suroccidental, se encuentran integradas en el tejido provincial. Por el contrario, estas localidades carecen de mecanismos y sistemas de ordenamiento espacial que promuevan una integración funcional como proyecto de desarrollo y que de paso resuelvan los conflictos que surgirán por el crecimiento promovido por las mejoras de las facilidades viales y las cercanías metropolitanas. Por ahora, el modelo tendencial no debería ser diferente a las urbanizaciones de baja calidad, a medio hacer y sin equipar, que se encuentran presentes en el perímetro metropolitano. Urbanizaciones de tipo suburbial que ya comienzan a extenderse en la comuna de Padre Hurtado.

Entonces, el modelo territorial vigente evidencia, dos fuerzas vectoriales:

El corredor metropolitano suroccidental en donde se emplazan de modo sobrepuesto, las principales entidades urbanas de la provincia, las facilidades viales, el trazado ferroviario, uno de los dos recursos fluviales de la provincia.

El núcleo de Isla de Maipo, que constituye un emplazamiento terminal y desvinculado de las facilidades viales y de dotación existentes en el corredor suroccidental de la provincia de Talagante.

Y dos conflictos:

Ausencia de un tejido vial provincial que refleje que el corredor metropolitano satisface las principales relaciones intercomunales y que predominan las relaciones de estas localidades con el espacio extraprovincial. La modificación del acceso libre de la Ruta 78 por una accesibilidad de pago en la Autopista del Sol, introducirá cambios en la configuración espacial y en el origen y destino de las relaciones de movilidad. Situación que puede incidir de modo positivo en una consolidación del sistema urbano provincial, a causa del aumento de los costos de desplazamiento.

Desequilibrios en la distribución poblacional. Sobre la franja subsidiaria del corredor suroccidental, se distribuye cerca del $90 \%$ de la población provincial. Porcentaje que 
con reparos puede ser aplicado a las actividades productivas, sociales y de dotación de servicios y equipamiento que presenta la provincia de Talagante. Situación que manifiesta desequilibrios y una gran presión de usos sobre una espacialidad reducida.

3.3.2. Objetivos y criterios de ordenamiento. Tal como ya lo hemos señalado en los dos informes anteriores, el objetivo fundamental de una política urbanística debe propender a que cualquier tipo de desarrollo de las entidades poblacionales vaya en provecho y beneficio directo de sus habitantes. Bajo tal filosofía, la Municipalidad debe transformarse en la protagonista del cambio, mediante el dominio de los bienes y plusvalías que promueva la formulación del instrumento de ordenamiento urbanístico. De este modo, el ente municipal se transforma en el motor del crecimiento, iluminando y orientando las iniciativas e intereses privados y concertando con éstos acciones dirigidas a corregir los conflictos funcionales y superar los déficits y carencias.

En función de ello, el modelo territorial propuesto debe resolver los conflictos que impiden la concreción el desarrollo razonable de la provincia de Talagante y potenciar sus atributos naturales y construidos. Los criterios de ordenamiento surgen como una respuesta al modelo tendencial y sustentan los principales postulados del modelo o proyecto territorial que se formula para la provincia de Talagante y la comuna de Isla de Maipo, de acuerdo al siguiente listado:

Articulación Espacial. El particular emplazamiento que registra la provincia, entre la aglomeración metropolitana y las facilidades portuarias y turísticas del litoral central, es el signo fundamental que determina el rol de la provincia en el espacio regional. Esta articulación debe asumirse y potenciarse mediante acciones precisas, con el fin de que el desarrollo y crecimiento promovido por esta cualidad articuladora comporte un mayor número de bienes y un menor número de conflictos, muchos de los cuales hoy constituyen trabas más que oportunidades de desarrollo.

Tejido Provincial. La transformación de la Ruta 78 en Ruta del Sol (de acceso libre la primera vía y acceso pagado, la segunda), divide la comuna en dos mitades y promueve una gran presión sobre las pocas vías transversales que unen ambas partes. A partir de ello, es necesario diseñar un tejido vial de accesibilidad y conexión provincial con el fin de corregir problemas derivados de la vialidad regional que cruza la provincia, los desequilibrios poblacionales y la existencia de territorios con trabas de conexión.

I dentidad Local. La introducción de mejoras en las conexiones viales, tanto metropolitanas como intercomunales, modificará la imagen de localidades rurales y agrícolas en ciudades satélites dormitorios de Santiago. El plan regulador debe promover la valoración de la identidad urbana y arquitectónica local, con el fin de evitar la pérdida de signos y valores diferenciadores.

3.3.3. El modelo provincial propuesto. De acuerdo a los datos consignados en los dos apartados anteriores, que tratan sobre la situación actual y los criterios de ordenamiento, el modelo propuesto se plantea como una respuesta a las limitaciones que presenta el modelo vigente, de neto carácter lineal y de dos dimensiones. La no intervención de este modelo promoverá la rápida transformación del espacio provincial según demandas provenientes de la aglomeración regional, siguiendo la misma ruta que ya han experimentado localidades emplazadas en el extrarradio rural y que hoy se encuentran anexionadas a causa de la expansión que caracteriza el crecimiento metropolitano. 
La aglomeración metropolitana crece de dos maneras: de modo lineal en las primeras fases de la expansión hacia suelos urbanos de nueva creación; la segunda fase, consolida de modo anular estos crecimientos. El resultado no puede ser distinto a los crecimientos vegetativos de la corteza de un árbol, siempre dependientes de una única centralidad y con pocos signos diferenciadores que potencien las identidades tradicionales de las localidades que se incorporan a la nueva espacialidad. Cuando surge un elemento diferenciado y excepcional, se manifiesta como un nudo espacial de difícil resolución.

Frente a estos desarrollos naturales o espontáneos de las ocupaciones antrópicas, proponemos un tejido ortogonal que equilibre las capacidades de acogida con las demandas productivas y urbanas. Los elementos constituyentes de este modelo territorial propuesto, son los siguientes:

- Creación de un tejido provincial. Este tejido integra los actuales sistemas vial y urbano, y las potencialidades productivas y los desarrollos inmobiliarios del suelo. En las zonas propiamente urbanas del corredor suroccidental (entre Padre Hurtado y Talagante), el trazado de este tejido está dimensionado por las facilidades viales existentes y en donde es posible promover usos urbanos y productivos de mayor intensidad. En las zonas de borde (correspondientes a las comunas de El Monte e Isla de Maipo), la dimensión del trazado responde a condicionantes de escala regional. Con ello se garantiza la protección de zonas de óptima capacidad de acogida agrícola y con mayores recursos paisajísticos y turísticos ${ }^{8}$.

- Interconexión vial entre el tejido ortogonal propuesto y el corredor suroccidental. La conexión entre el nuevo tejido ortogonal y la vialidad regional existente permite la accesibilidad en puntos extremos, asegurando con ello una mayor integración entre el corredor de alta velocidad y el sistema urbano provincial.

- Triangulación del territorio. El diseño del tejido ortogonal promueve una ocupación racional del territorio, alternativa a la ocupación de naturaleza lineal y bidimensional que sustenta el corredor suroccidental y la franja de ocupación urbana y productiva que éste promueve. La incorporación de nuevas zonas e integración de núcleos aislados, tales como los que se observan en la comuna de Isla de Maipo, triangula el espacio y permite considerar la superficie útil de la provincia con similares planteamientos de sustentabilidad.

- Multiplicación de las centralidades. La configuración de una trama o tejido ortogonal, opuesto a las configuraciones radiales y anulares que se asientan en la unicentralidad y en el permanente surgimiento de periferias (efecto árbol), transforma cada vértice de esta cuadrícula en una centralidad urbana. Por ello, es de fundamental importancia que este tejido reconozca la vialidad existente.

\footnotetext{
${ }^{8}$ Esta propuesta de armadura provincial se diferencia de modo fundamental de la propuesta de Pedro ORTIZ y Francisco de CASO, 1998, (Santiago de Chile, Ordenación reticular del territorio (ORT) 1998-2028. Comunidad de Madrid, Consejería de Obras Públicas, Urbanismo y Transporte, Dirección General de Urbanismo y Planificación Regional. 32 págs. y 3 planos), la que propone la ordenación reticular del territorio mediante la definición de una malla o retícula que se sobrepone en el espacio regional de forma homogénea, justamente por esto último. En nuestro caso particular, consideramos que el territorio no es una dimensión homogénea por el hecho de existir una estructura natural históricamente plasmada en las relaciones urbanas que se establecen a partir de grados de influencia y dependencia entre los diferentes centros urbanos de la región metropolitana. Por ello, la armadura propuesta se inserta en la estructura histórica de escala provincial existente, la completa y la articula con la estructura metropolitana y con las otras estructuras provinciales de la región.
} 
- Nuevos desarrollos inmobiliarios. Frente a los nuevos usos urbanos del tipo parcelas de agrado y condominios en las áreas de alta calidad agrícola y paisajística, la ortogonalización del territorio promueve áreas o puntos precisos con mejores facilidades viales y de urbanización. Con ello se promueve la protección de zonas con buenas capacidades de acogida agrícola, paisajística y ambiental. La consideración de los pequeños núcleos como vértices del tejido ortogonal, también motiva un ordenamiento urbano alternativo del territorio. (Ver Fig. 4).

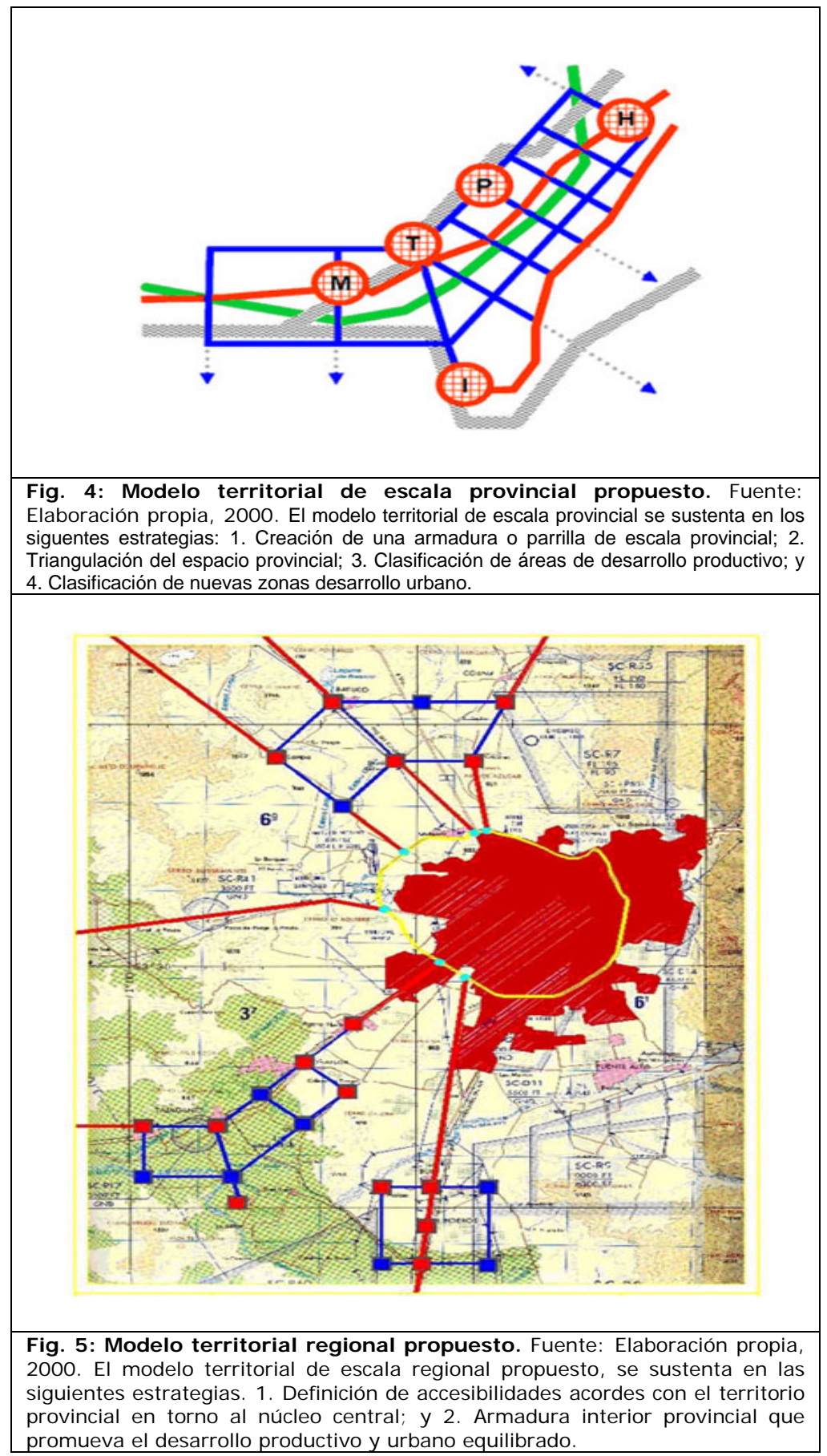


4. A modo de conclusiónGran parte de las entidades provinciales y comunales del país, carecen de una estructura propia que ordene la ocupación del territorio y se constituya en el soporte de los diferentes sistemas que convergen sobre un mismo espacio. Articular, estructurar, integrar, ordenar y utilidad constituyen el abecedario básico del ordenamiento territorial.

La modificación del Plan Regulador Metropolitano de Santiago de 1994, asentado sólo en la extensión una vez más del continuo urbano, nos presenta la oportunidad para reflexionar acerca del modelo territorial, sus conflictos y oportunidades para repensar las metodologías y estrategias que es necesario aplicar para optimizar los resultados de las actuaciones públicas en el territorio.

En términos más amplios, las complejidades que impone la geografía del país sobre los sistemas que comparecen en el espacio físico, nos llevan a traducir esta reflexión en los objetivos básicos del ordenamiento y del proyecto territorial, de acuerdo con el siguiente abecedario:

A. Articular. En los últimos años, hemos asistido a un interesante renacer de visiones territorialistas de parte de organismos con competencias específicas en tales materias. El problema surge cuando estas visiones se formulan de modo unilateral, con escasa compatibilidad con otras actuaciones que convergen en una misma espacialidad. Si ninguna disciplina en particular tiene instrumentos para operar sobre todo el territorio, tampoco el territorio puede ser reducido a una sola y particular visión, sea ésta ambiental o urbanística, dejando de lado otras facetas o dimensiones. En tanto visión mayor y expresión física y administrativa de las políticas sectoriales, los instrumentos de ordenamiento territorial son los llamados a resolver las incompatibilidades y ordenar los resultados obtenidos parcialmente.

E. Estructurar. Parte de los conflictos que se expresan en dificultades o conflictos para los sistemas productivos o sociales, por citar algunos, tienen su origen en la ausencia de una estructura territorial de escala provincial y comunal. La totalidad de las dimensiones administrativas del país carece de una propia armadura o esqueleto básico, en donde insertar el patrón de asentamientos urbanos y el sistema productivo, por ejemplo. Esta falta de estructura deja en evidencia no pocas veces, la incompatibilidad que existe entre el territorio y los sistemas que operan sobre él, provocando conflictos y deterioros. Valga la imagen de la propuesta de armadura territorial de la provincia de Talagante consignada en este escrito, para ejemplarizar lo que entendemos como una estructura espacial.

I. Integrar. Por su forma física, la estructura territorial del país carece de razón si no hay una integración fronteriza con los países vecinos. Es más, las posturas que se adopten para ordenar los usos del territorio deben pasar por el diseño de estructuras de integración transfronteriza. Los ramales ferroviarios construidos entre los siglos XIX y $\mathrm{XX}$, tuvieron desde siempre una vocación transandina que es menester recuperar. Frente a las propuestas argentina y boliviana de los trazados ferroviario transpatagónico y del gasoducto, el país debe tener su propio proyecto con el fin integrar y optimizar estas acciones foráneas.

O. Ordenar. El exacerbado centralismo que exhibe la escala nacional, se reproduce en los niveles regional, provincial y comunal. Esta cuestión deteriora las capacidades de acogida del territorio, a veces por congestión de usos y otras, por la infrautilización de los valores del mismo. En estos casos, se echa en falta una directriz que ordene y jerarquice la intervención de los niveles que operan sobre el territorio. 
U. Unificar. Ya no es posible seguir actuando de modo sectorial, desperdiciando las posibilidades que se nos presentan cuando disponemos de una pre - visión que supere las limitaciones sectoriales. Ello sólo es posible mediante la formulación de un proyecto territorial acorde con las características regionales y sus capacidades de acogida, en cuantos factores primordiales del proceso de desarrollo. A estas alturas, ya no podemos seguir instalando cualquier cosa en cualquier parte. Por el contrario, diseñar el territorio debe ser la acción básica para mejorar la calidad de vida de la sociedad.

\section{Referencias}

- CHILE, MINISTERIO DE OBRAS PÚBLICAS, Dirección de Planeamiento, Departamento de Planes de Obras Públicas, BRIEVA ALVARADO, Amador y GONZÁLEZ MONTECINOS, Ventura [autores en 1958], La Unidad Micro-Regional: características y métodos para delimitarla. Santiago de Chile, Publicación N6, junio de 1962.

-CIDU, Equipo Macrozona Central, “Síntesis del estudio 'Región central de Chile: perspectivas de desarrollo' (1971-1972)", en: de MATTOS, C., FIGUEROA, O.; BANNEN, P.; CAMPOS, D. /Editores) Santiago en EURE: Huellas de una metamorfosis metropolitana 1970/2000, I.E.U.y T., P. Universidad Católica de Chile, 545 págs.

-ORTIZ, Pedro, CASO, Francisco de. 1998. Santiago de Chile, Ordenación reticular del territorio (ORT) 1998-2028. Comunidad de Madrid, Consejería de Obras Públicas, Urbanismo y Transporte, Dirección General de Urbanismo y Planificación Regional. 32 págs. y 3 planos. Versión digital in extenso con ilustraciones a color [en línea]: http://urbanismo.8m.com/lecturas/reticula.pdf

-PARROCHIA B., Juan (autor de contenidos) M.I.PAVEZ R. (propósitos y comp.), El Plan Tridimensional de Ordenamiento Territorial y la Región Metropolitana de Santiago 1960-2000. Ed. D. Urbanismo, F.A.U. U.CH. 1a edición, impresa b/n, nov. 1994, 216 págs., contiene selección de planos originales. 2a edición, versión digital in extenso, revisada y ampliada en 2001, ilustraciones color,[en línea]:

http://mazinger.sisib.uchile.cl/repositorio/lb/arquitectura_y_urbanismo/pavezmi01

-PÁVEZ REYES, M. Isabel: "Identidad, ordenamiento territorial y oportunidades: espacios públicos y recreación para la Región Metropolitana de Santiago Siglo XXI", en REVISTA DE URBANISMO, digital, N¹4, D. Urbanismo F.A.U. U. de Chile, Primer sem. 2006, 21 págs. Ilustradas, [en línea]: http://revistaurbanismo.uchile.cl

-PÁVEZ REYES, M. Isabel. 2006. Vialidad y Transporte 1950-1979 en Santiago de Chile: Concepto y Estrategia de Ordenación del Territorio en el marco de la Planificación Urbana-Regional por el Estado de Chile. Tesis Doctoral en Arquitectura y Urbanismo, Universidad Politécnica de Madrid, Director Dr. Ing. Julio Pozueta E., 13 de diciembre de 2006.

-SERRANO RODRIGUEZ, Antonio, “EI modelo territorial europeo: tendencias para el siglo XXI y sus implicaciones para el modelo territorial español", en revista URBAN N ${ }^{\circ} 8$ Madrid, editada por la ETSAM - UPM, otoño 2003, pp. 35 - 54. 